\title{
Peculiarities of the Reproductive Health of Women Affected by Hepatobiliary Disorders
}

\section{Oleksandra Boychuk ${ }^{1}$ and Nsan Ekom Nsed Ebaye ${ }^{2 *}$}

${ }^{1}$ Professor at the Subdepartment of Obstetrics and Gynecology of the Department of Postgraduate Studies of Ivano-Frankivsk National Medical University, Ukraine ${ }^{2}$ Bakor Medical Center, Cross River State, Calabar, Director of Clinical Services, Nigeria

*Corresponding Author: Nsan Ekom Nsed Ebaye, Bakor Medical Center, Cross River State, Calabar, Director of Clinical Services, Nigeria.
Received: November 01, 2021

Published: November 30, 2021

(C) All rights are reserved by Oleksandra

Boychuk and Nsan Ekom Nsed Ebaye.

\begin{abstract}
The World Health Organization considers the protection of reproductive health to be a priority area of its activities and calls on all countries to ensure further development of programs promoting reproductive health [1].

In 2006, the UN General Assembly adopted are commendation to include the goal of achieving general access to reproductive healthcare in the Five Millennium Development Goals. Environment, working conditions, social aspects of people's life, mental condition, somatic disorders - all these factors influence the functional condition of the reproductive system.
\end{abstract}

Keywords: Peculiarities; Reproductive Health; WHO

\section{Introduction}

One of the indicators of the condition of reproductive health is infertility. An infertility rate of 10 to $20 \%$ may be regarded as direct reproductive loss [2]. The WHO is concerned about the fact that at the beginning of the $21^{\text {st }}$ century, infertility may become the third most prevalent disease after oncological pathology and cardiovascular diseases.

Infertility is widely spread all over the world. For many years, infertility rates in various regions of the world have ranged from 8 to $18 \%$ and there is no downtrend in sight. Nowadays over 80 million people in the world are believed to be affected by infertility [3]. According to the European Society of Human Reproduction and Embryology, about $10 \%$ of married couples in Europe are in fertile and in the USA, this rate is from 10 to $15 \%$.

According to the official statistics, the infertility rate in Ukraine is not high - from 2.8 to 3.5 per 1,000 women and from 0.3 to
0.4 per 1,000 men. However, sociological surveys reveal that undesired infertility affects from 6 to $20 \%$ of families in various regions of Ukraine [4].

In recent years, there has been an increase in somatic and gynecological morbidity levels among women all over the world, which has a negative impact on the realization of the reproductive function [5].

The development of efficient methods of treatment of female infertility is one of the top priorities of the contemporary gynecology, which is accounted for, on the one hand, by high female infertility rates and, on the other hand, by a wide variety of infertility factors. If women follow all the recommendations from medical specialists, the conception rate ranges from 10 to $89 \%$ and depends on reasons causing reproductive function disorders. In the situations when there is no effective treatment result, it is worth considering the use of assisted reproductive technologies (ART) [3]. Specialists 
have proved that the cumulative efficiency of ART corresponds with natural fertility and makes up from 25 to $30 \%$ for a cycle. Alongside with absolute indications for ART and depending on the condition of the ovarian reserve, these methods are first of all indicated for women older than 35 years [6].

In recent decades, assisted reproductive technologies have made a long way, accumulating innovative scientific achievements and the most revolutionary ideas of the contemporary biology. According to CDC, in the period from 2001 to 2010, the percentage of embryo transfers resulting in the birth of healthy babies in the USA increased by 2 to 7\%. The ESHRE registered approximately the same ART statistics in Europe [7].

Alongside with the increase in the number of highly specialized reproductive clinics, the reduction of the cost of treatment and the improvement of its accessibility to patients, one of the persisting challenges is the improvement of the efficiency of ART programs and the reduction of risks. It is traditionally believed that the efficiency of ART programs is influenced by a couple's age, the parameters of sperm and ovarian reserve, the presence of a concomitant pathology and positive medical history.

In the last decades, women have experienced significant changes in their social life, which has led to the postponement of the birth of children to a later period in life, when women's capabilities are limited to this or that extent. There is a number of medical aspects making reproduction more difficult in the advanced maternal age, including the state of somatic and gynecological health of women, the functional aging of the reproductive system, etc.

New approaches to ART are characterized by the beginning of treatment long before the first injection of hormones. So far, the preconception preparation of couples seeking treatment of infertility has been sporadic and poorly structured. Now that there is a clear understanding of the negative effects of various factors (e.g. medications, household chemical goods, nicotine, alcohol or other psychotropic drugs) on the quality of sex cells, implantation, pregnancy and state of health, ART program providers make wider use of a prophylactic approach to the optimization of the conception conditions.

Thus, the state of a woman's reproductive health is influenced by various factors, among which special importance is attached to the presence of somatic diseases, in particular hepatobiliary pathology. This is currently recognized by many scientists, yet the specific mechanisms are scarcely researched and not fully identified. There are research data on the influence of hepatic pathology on the effectiveness of ART for the treatment of infertility. On the other hand, there are data on the negative effect on the liver of hormonal and other therapies used in the course of ART.

\section{Discussion}

In the last 20 years, there has been a clear growing trend throughout the world in the hepatobiliary morbidity. Hepatobiliary pathologies have become more frequent among young people, with women being 4 to 7 times more often affected than men. According to the WHO experts, every fifth woman in Europe suffers from a hepatobiliary pathology [8].

The hepatobiliary system, which includes the liver itself, bile ducts and the gallbladder, is involved in many processes of vital activity of a human body. Its lesions cause serious disorders of metabolism, immune response, detoxification and antibacterial defense. The liver is actively involved in the adaptive reactions to the diseases of other organs and systems. At the same time, functional insufficiency of the liver because of its lesion may lead to a severer course of pathological processes in other organs, change hormonal regulation or influence the effectiveness of a drug therapy [9].

The liver participates in the metabolism of a number of hormones, that's why chronic liver diseases may go together with hormonal disorders. On the other hand, estrogens may directly disturb the liver's secretory function. Estradiol and other estrogens (for example, those contained in oral contraceptives) influence the release of bile acids and deteriorate the disorders of direct bilirubin production in patients affected by Dubbin-Johnson syndrome. They also cause an increased activity of alkaline phosphatase in serum. The central role in the system of hepatic microsomal metabolism of female sex hormones and their analogues is played by cytochrome P450 isoenzymes (CYP-450) [10].

One of the effective ways of the body's adaptation to pathological disorders in the release of hormones lies in the regulation of hormone binding by proteins in blood. The neutralization of the adverse impact of hypo- and hypersecretion of hormones is also achieved by the changes in the activity of enzymes of the liver and other organs where these hormones are destroyed and inactivated. 
Liver cells are most often damaged by aggressive compounds - poisons, free radicals, bacterial and viral infections. Besides, destructive influence on the liver comes from stress, hormonal and metabolic disorders, food of bad quality, adverse effects of medications and alcohol [9].

In the pathogenesis of liver lesions of various etiology, two types of pathological reactions should be noted:

- Direct liver lesion by an etiological factor (viruses, chemicals, medications, blood flow disorders), which manifests itself in dystrophic changes up to necrosis;

- $\quad$ autoimmune liver lesion by autoantigens (pathologically changed hepatocyte components that develop due to direct liver lesion) and auto allergic reactions of humoral and cellular types. The latter deteriorate liver lesion because of microcirculation disorders and immune cytolysis by killer T-cells.

Liver lesion may lead to disorders of all types of metabolism: carbohydrate metabolism, lipid metabolism, protein metabolism, metabolism of vitamins, hormones, biologically active substances and microelements.

Disorders of metabolism of hormones and biologically active substances in cases of liver pathology manifest themselves in changes in the synthesis of hormones, their transport proteins; inactivation of hormones; inactivation of biologically active substances.

Liver lesion by a pathological process causing disorders of inactivation of such hormones as thyroxin, insulin, corticosteroids, androgens and estrogens leads to deviations in their concentration in blood and the development of endocrine pathology.

Recently special attention has been devoted to the genetic factors of the development of liver pathology. There have been reports of a significant role of genetic factors disturbing the immunological tolerance to autoantigens in the development of immunologically determined liver lesions [3,14-18].

Estrogenmetabolismisalsodisturbedunderthecombinationofadverseexternalfactorsandgeneticpredispositiontoit $\quad[12,14]$, which is determined by polymorphism of genes encoding the enzymes of the first and the second phase of detoxification (CYP 1A1,
CYP 1B1, CYP 3A4, COMT, MTHFR, GST, etc.) [10].

Recent studies have identified a number of gene polymorphisms associated with the development of drug-induced liver injuries (DILI). Two main categories of genes are distinguished: highly polymorphic genes of the major histocompatibility complex (HLA), which determine the individual peculiarities of the immune response to one and the same drug, and polymorphic genes encoding enzymes and transport proteins and determining the individual peculiarities of drug metabolism, susceptibility to cholestasis [11].

One should note the importance of genetic and hormonal factors in the development of intrahepatic cholestasis of pregnancy (ICP). One has found a clear relation between the known cholestasis genes like ABCB4 (MDR3), ABCB11 (BSEP) andATP8B1 (FIC1) and ICP.

The use of methods of proteomics, one of the most promising areas of development of medicine and biology, contributes to the identification of protein biomarkers that can potentially change the strategies of early diagnosis and treatment of a number of diseases. The majority of the proteomic tools may also be used for the study of physiological and pathological events related to reproductive biology. Studies are underway regarding the opportunities of proteomics for the research of mechanisms of disorders of the human reproductive system like spermatogenesis, oogenesis, endometriosis, polycystic ovary syndrome, embryo development. The objective is to use new technologies like2-DE, Dige, MALDIMS, SELDI-MC, MUDPIT, LC-MS, etc. for the understanding of the molecular aspects of reproduction in normal and pathological conditions [12].

The interrelation between the reproductive system and the hepatobiliary system has been known for quite a long time [13]. Close functional relations between the condition of the liver and female sex hormone levels, concomitance of their disorders as well as the necessity to apply a hormonal therapy that influences the liver activity account for an increased interest for the study of the influence of the functional condition of the liver on women's reproductive health $[3,13]$.

The key role of the liver in fertility has been proved by several foreign studies in recent years. One of the latest studies [14] has revealed that estrogen receptors in the liver are crucial for the 
maintenance of reproduction. Moreover, the expression of these receptors is controlled by essential amino acids, the building blocks of proteins. The findings of the experiments may have important consequences for certain forms of infertility. These studies were the first to prove experimentally the importance of the liver for reproduction. The idea of a diet influencing fertility is not new but this explains how a diet, especially a low-protein diet, may have a direct impact. The authors report that the expression of estrogen receptors depends on essential amino acids. A low-calorie diet in case of lack of estrogen receptors in the liver leads to the reduction of the level of the IGF-1hormone in blood to a level that is inadequate for a proper gestational development. When more proteins were added to the diet, the reproductive cycles were restored. On the other hand, food fats and carbohydrates had no effect on estrogen receptors or reproduction. The scientists presume that this relation between amino acids, estrogen receptors in the liver and the reproductive function may have clinical consequences. For example, this may explain why people suffering from anorexia are usually infertile. This suggests that diets containing mainly carbohydrates and low quantities of proteins may hinder reproduction.

Other experimental studies [15] highlight the role of Lipin-1 in the regulation of estrogen in the two major target organs (uterus and liver) and suggest new prospects for these lipids in the regulation of phosphatase not only in the central metabolism regulation but also in the function of the uterus and reproductive biology. The authors [16] consider the role of liver X receptors (LXR) in the reproductive system and the prospects of use of LXR agonists in the treatment of reproductive pathologies.

Estrogens, gestates and their contemporary synthetic analogues (homologues) are widely used as medications in the obstetrical and gynecological practice for various pathologies related to the insufficient hormonal function of ovaries; menstrual disorders; infertility; for the correction of labor activity; hormonal contraception. Hormonal therapy may also be prophylactic. Although the progress in the pharmaceutics of hormonal drugs has led to the introduction in the clinical practice of a wide range of medicinal estrogens and gestagens with minimal risks of adverse side effects, the problem of safety of hormonal therapy with medicinal estrogens and gestagens remains, in particular a serious risk of their hepatotoxic and thrombotic (thromboembolic) effect [10].
Potential structural and functional biochemical disorders in the course of hormonal therapy become more serious in case of presence of hepatobiliary diseases or susceptibility to them. Therefore, before beginning any hormonal therapy, including within the framework of ART, it is necessary to carry out a screening to identify potential contraindications to the use of estrogens and gestagens, including genetic predisposition.

Besides, the change of the liver's synthetic activity under the influence of estrogens may lead to a prognostically unfavorable estrogen-induced prothrombotic, vein-occlusive and thromboembolic reactions (increased risk of venous thrombosis and thromboembolism) in the vascular system of the liver up to a rare (but highly lethal) portal vein thrombosis.

The most typical and predicted hepato pathic effects of mainly enteral forms of medicinal estrogens and gestagens are usually related to the risk of the development of cholestasis (with increased bile litho genicity and cholelithiasis) and noninflammatory hepatocyte injury. The risk of the development of intrahepatic cholestasis without necrosis and hepatotoxic effect in case of the use of estrogens and gestagens (the latter increase the toxicity of the former) increases in case of jaundice in the medical history during pregnancy.

According to the regulation on the use of assisted reproductive technologies adopted by Order No. 771 of 23.12.2008 of the Ministry of Health of Ukraine, the following groups of medications may be used for controlled stimulation of ovaries: selective estrogen receptor modulators (SERM), aromatase inhibitors, gonadotropins: human menopausal gonadotropin (hMG), follicle-stimulating hormone (FSH); recombinant medications: recombinant follicle-stimulating hormone (rFSH), recombinant luteinizing hormone ( $\mathrm{rLH})$; chorionic gonadotropin (CG); gonadotropin-releasing hormone (GnRH) agonists; gonadotropin-releasing hormone (GnRH) antagonists. Thus, in such women, there is a high likelihood of adverse effects of medications on the liver, which accounts for the necessity of screening for hepatobiliary diseases and susceptibility to them before the therapy start and of the examination of the liver condition after it, especially in case of pregnancy.

The issue of "liver diseases and pregnancy" has been the object of many national and foreign studies, although most of them 
describe the clinical aspects of the problem and the specific pathogenetic mechanisms of obstetrical and perinatal disorders remain not fully identified. The issues of the conditions of achieving and maintaining pregnancy, the course and perinatal aspects of pregnancy in women affected by hepatobiliary pathology and in need of ART are practically not addressed, in particular, there is no research on the genetic predisposition to disorders, the influence of drug therapy, etc.

One singles out two groups of liver diseases concomitant with pregnancy: pregnancy-related and not pregnancy-related diseases [17].

In English sources, one can find 3 groups of diseases [18]:

- $\quad$ Diseases existing before pregnancy (chronic hepatitis type $\mathrm{B}$ and $\mathrm{C}$, auto immune hepatitis, Wilson-Konovalov disease, primary biliary cirrhosis, primary sclerosing cholangitis, cirrhosis);

- $\quad$ Diseases unique to pregnancy (earlygestationaltoxicosis, intra hepatic cholestasis of pregnancy, acute fatty liver of pregnancy, pree clampsia and eclampsia, HELLPsyndrome);

- $\quad$ Diseases detected in the course of pregnancy (acute viral hepatitis, cholelithiasis, Budd-Chiari syndrome, drug-induced hepatotoxicity, sepsis).

Thereareanumberoftheoriesofpathogenesisofthedevelopmentofpregnancy-related liver diseases, including the hormonal, the genetic and other theories. According to the hormonal theory, an increased release of progesterone and other placental hormones in a pregnant woman's body inhibits the release of gonadotropic hormones by the hypophysis and leads to an increased cholesterol synthesis in the liver and a decreased capacity of the membranes of hepatocytes and bile canaliculi to release bile acids. Pregnancy only reveals the existing genetic defects. A disorder of estrogen and progesterone sulfation in the liver and the deterioration of the draining function of bile canaliculi are a hereditary combination of two defects. Great importance is attached to the mutation of proteinencoding genes - bile acid transporters (MDR3, BSEP, MPP2) [19].

According to the contemporary principles of drug therapy of liver diseases, the program of complex treatment of such pathology includes several major lines [20]:
- Prophylactic therapy aimed at ensuring the primary protection of the liver from lesion;

- $\quad$ Etio tropic therapy aimed at suppressing the pathogen, its elimination and sanitation of the body;

- Pathogenetic therapy aimed at an adequate pharmacological correction of universal multi-factor and noncontemporaneous components of disease pathogenesis. It should be noted that, in spite of the polyetiological nature of liver lesions, certain similarity of the main components of pathogenesis allows the use of a rather similar pathogenetic therapy;

- Symptomatic therapy.

Among the medications used for the treatment of liver diseases, one may single out a relatively small group of medications in which the hepatotropic effect is predominant has an independent clinical significance. They are traditionally referred to as hepatoprotective drugs. The expected effect of these drugs is aimed at restoring homeostasis in the liver, improving the organ's resistance to the influence of pathogenic factors, normalizing the functional activity and stimulating the reparative and regenerative processes in the liver.

The prevention of adverse side effects of hormonal therapy should be based on the identification of patients' individual contraindications to it, personalized prognostication of possible adverse effects, laboratory and instrumental examination of patients, minimization of simultaneous prescription of hepatotropic drugs and exclusion of hepatotoxic xenobiotics. If there is choice, one should give preference to parenteral (local, transdermal) ways of administering estrogens and gestagens.

Diet therapy, mineral water and other non pharmaceutical means reduce the adverse effects of hormonal therapy on the hepatobiliary system.

The additional use of single and combined herbal remedies with choleretic action (immortelle, barberry, corn silks, yarrow, peppermint, artichoke, Liv. 52, etc.) and liver metabolism correctors (inosine, methionine, lipoic acid, etc.)also contributes to the optimization of the figures of adverse hepatocellular effects of estrogens and gestagens. 
It should be noted that the use of contemporary estrogen and gestagen hormonal drugs still implies a certain risk of adverse effects on the hepatobiliary system. Although the hepatotoxicity of estrogens and gestagens is more likely when alterations in the activity of the hepatobiliary system already exist before the hormonal therapy, deviations from the reasonable dosage and a simultaneous use of xenobiotics that deteriorate liver metabolism and clearance of estrogens and gestagens may have a negative impact on the liver condition. The reasonability and the strategy of further hormonal therapy with estrogens and gestagens in cases of adverse effects are specified individually, taking into consideration their necessity, the prognosis of the main disease, and after informing patients about the possible adverse consequences of hormonal therapy. A potential risk of hepatotoxicity, cholestasis and other adverse effects of medicinal estrogens and gestagens must not exceed the expected benefit from hormonal therapy.

\section{Conclusion}

We believe that it worthwhile researching the hormonal and metabolic disorders in patients with infertility and hepatobiliary pathology, developing methods of preliminary preparation for ART, detecting and correcting genetic factors that have a negative impact on the prognosis of conception and the outcome of pregnancy, optimizing the patterns of induction of superovulation and the methods of management of early gestational stages, which will enable the improvement of efficiency and safety of the use of ART in this category of patients and reduce to a minimum the risk of adverse effects on the hepatobiliary system.At present, there is an uncontestable necessity of establishing new genetic and proteomic markers of predisposition to various pathological conditions, in particular hepatobiliary diseases, which determine the success of the use of ART and the perinatal consequences of such pregnancy.

\section{Bibliography}

1. WHO Reproductive Health Strategy (2004).

2. Yuzko OM., et al. "Assisted reproductive technologies in Ukraine: pregnancy, labor, newborns". Women's Doctor 4 (2013): 52-53.

3. Ovsiannikova TV., et al. "Contemporary principles of diagnosis and treatment of infertility". Gynecology 4 (2009): 30-33.

4. Yuzko OM., et al. "Assisted reproductive technologiesin Ukraine". Women's Doctor 3 (2007): 8.
5. VI Kulakov., et al. "Structure of female infertility and prognosis of recovery of the reproductive function at the use of the contemporary endoscopic methods". Obstetrics and Gynecology 3 (2001): 33-36.

6. Mishiyeva NG. "Infertility in women of advanced maternal age: principles of diagnosis and treatment depending on the ovarian r: abstract of MD dissertation". Moscow (2008): 47.

7. Quality clinical treatment with the use of assisted reproductive technologies. ESHRE program document. Access mode (2008).

8. Skrypnyk IM. "Clinical hepatology". Poltava: Dyvosvit (2007): 425.

9. Podymova SD. "Liver diseases". $4^{\text {th }}$ edition, revised. Moscow: Meditsyna (2005): 704.

10. Karachentsev AN. "Hepatobiliary system as target for adverse effects of medicinal estrogens and gestagens". Russian Bulletin of Obstetrics and Gynecology 6 (2004): 20-25.

11. RA Wilke., et al. "Identifying genetic risk factors for serious adverse drug reactions: current progress and challenges". Nature Reviews Drug Discovery 11 (2007): 904 -916.

12. Upadhyay RD., et al. "Proteomics in reproductive biology: beacon for unravelling the molecular complexities". Biochimica et Biophysica Acta 1834.1 (2013): 8-15.

13. Dubossarskaya YA. "Hepatobiliary pathology in gynecological practice". Medical Aspects of Women's Health 3.30 (2010): 1219.

14. TS Della., et al. "AminoAcid-Dependent Activation of Liver Estrogen Receptor Alpha Integrated Metabolic and Reproductive Functions via IGF”. Cell Metabolism 13.2 (2011): 205-214.

15. PM Gowri., et al. "Lipin1 Regulation by Estrogen in Uterus and Liver: Implications for Diabetes and Fertility". Endocrinology 148.87 (2007): 3685-3693.

16. Bełtowski J "Liver X receptor (LXR) and the reproductive system - a potential novel target for therapeutic intervention". Pharmacological Reports 62.1 (2010): 15-27.

17. Ignatova TM. "Liver diseases in pregnant women". Medical Bulletin of the North Caucasus 2 (2009): 88-93. 
18. Bacq Y. "Liver diseases unique to pregnancy: a 2010 update". Clinics and Research in Hepatology and Gastroenterology 35 (2011): 182-193.

19. OI Fediv., et al. "Peculiarities of etiology and pathogenesis of intrahepatic cholestasis of pregnancy". Clinical and Experimental Pathology 12.1 (2013): 197-200.

20. Kramarev SA "Contemporary hepatoprotective drugs in hepatology". Pediatrician 1 (2011): 5-9.

Volume 4 Issue 12 December 2021

(C) All rights are reserved by Oleksandra Boychuk and Nsan Ekom Nsed Ebaye.

Citation: Oleksandra Boychuk and Nsan Ekom Nsed Ebaye. "Peculiarities of the Reproductive Health of Women Affected by Hepatobiliary Disorders". Acta Scientific Gastrointestinal Disorders 4.12 (2021): 49-55. 